\title{
KIT EDUCACIONAL PARA CONTROLE E SUPERVISÃO APLICADO A NÍVEL
}

\author{
Jonathan Paulo Pinheiro Pereira \\ Engenheiro de Computação - UFRN 2005, Especialista em Engenharia Elétrica - UFRN \\ 2008. E-mail: jonathan@cefetrn.br
}

Ricardo Alexsandro de Medeiros Valentim

Chefe do Departamento de Pesquisa e Inovação - IFRN / Natal Campus Central

Doutor em Engenheiro de Computação

E-mail: valentim@cefetrn.br

Bruno de Paiva e Silva Castro

Estudante de Automação Industrial - IFRN

E-mail: brunogessinger@hotmail.com

\section{RESUMO}

Visto a necessidade de ferramentas didáticas para utilização em aulas práticas nas disciplinas de Controle de Processos e Sistemas Supervisórios e também devido os elevados preços desses equipamentos no mercado especializado, propôs-se o projeto e implementação de um mini kit didático, aplicado a variável nível, com objetivo principal de suprir essa deficiência. Na indústria automatizada, sistemas de supervisão e controle são muito presentes, podendo ser encontrados nas mais diversas aplicações, como por exemplo: sistemas para controle térmico de caldeiras, supervisão e controle de vasos de pressão e controle de nível de reservatórios de petróleo. Neste contexto, este artigo apresenta um kit que foi confeccionado para servir de instrumento nas aulas práticas de controle e sistemas supervisórios, possibilitando, portanto uma maior interação do aluno com a prática.

PALAVRAS-CHAVE: Controle, Supervisório, Kit Didático.

\section{EDUCATIONAL KIT FOR CONTROL AND SUPERVISION APPLIED TO LEVEL}

\begin{abstract}
:
Given the need of educational tools for utilization in disciplines of the Process Control and Supervisory Systems and having in mind them elevated prices of those equipments in the specialized market, proposed the project and implementation of a educational kit applied the variable level, with main objective of supply this deficiency. In the automatic industry, systems of supervision and control are much present, being able to be found in the most diverse application, as by example: thermal systems control of boilers, supervision and pressure control and oil reservoirs level control. The project has for purpose the implementation of a kit that will serve of instrument in the disciplines of control and supervisory systems, enabling a bigger interaction of the student with the practical.
\end{abstract}

KEYWORDS: Control, Supervisory, Educational Kit. 


\section{KIT EDUCACIONAL PARA CONTROLE E SUPERVISÃO APLICADO A NÍVEL}

\section{INTRODUÇÃO}

A determinação do nível e o seu controle são pontos bastantes comuns observados em processos industriais, por exemplo, nas indústrias químicas, petróleo, papel, alimentos, têxtil, tratamento de água e no tratamento de efluentes. Isso porque, envolvem o controle operacional ou controle de custo. O nível de líquido é, em geral, expresso como uma medida de comprimento em relação a uma referência (base de um tanque, por exemplo).

No processo de abastecimento de água, os reservatórios têm como principais funções operar como reguladores da distribuição, atendendo à variação de horária do consumo. $\mathrm{O}$ objetivo desta operação é assegurar uma reserva d'água para combate incêndio, criando, portanto, uma contingência para atender as condições de emergência, atender à demanda no caso de interrupções de energia elétrica e a manutenção de pressões na rede distribuidora.

Geralmente os reservatórios demandam controle de nível a fim de mantê-lo cheios de forma a não extravasarem. Os sistemas mais comuns são os de bóia ligados a sinaleiros, ou a controles elétrico-eletrônicos de comando das bombas, que são utilizados no abastecimento desses. Neste contexto, existem também, os sistemas de aquisição de dados que são utilizados com a finalidade de obter as informações necessárias para o bom funcionamento do sistema, visto que permite realizar o processamento desses dados, e gerar processos de atuação quanto ao controle de nível.

Nos cursos técnicos e tecnológicos oferecidos pelo Instituto Federal de Educação, Ciência e Tecnologia (IFRN) nas áreas da indústria, tais como, Tecnologia em Automação Industrial, Técnico em Petróleo e Técnico de Mecânica, grande parte das disciplinas de instrumentação e controle de processos é ministrada em boa parte de forma teórica. Está característica define, portanto a necessidade de uma ferramenta didática, prática e portável para simulação de plantas de processos industriais, bem como a implementação e ajuste de controladores.

Diante desta perspectiva, este artigo apresenta um mini-kit didático, que foi desenvolvido para preencher a lacuna das aulas teóricas. Neste sentido, melhorar em aspectos didáticos as disciplinas de controle e controle de processos, uma vez que o mini-kit contribui no desenvolvimento das aulas práticas, que são essenciais para os cursos na área da indústria.

\section{DESCRIÇÃO DO KIT}

O modelo didático é formado por um conjunto de dois reservatórios montados um sobre o outro. O Elemento responsável pela interface PC-PLANTA é um CLP (Controlador Lógico Programável). O controle é aplicado para regular, segundo um valor de referência, o nível do reservatório superior. As informações utilizadas na realimentação do sistema são obtidas por um sensor potenciométrico acoplado a um sistema de bóia. Para supervisão foi utilizado uma aplicação desenvolvida no software INTOUCH ${ }^{\circledR}$. Como elemento final de controle foi utilizado uma bomba alimentada por uma fonte ATX de PC, que faz uso da técnica de modulação por largura de pulso. 
O funcionamento do mini-kit tem o seguinte fluxo descrito a seguir, e na Figura 1 é possível ter uma visão geral de todos os elementos do kit, bem como, de suas dependências:

$0 \quad$ O sistema microcontrolado recebe uma informação analógica do CLP;

1 A informação é convertida em uma onda quadrada de ciclo ativo variável;

2 A onda é aplicada ao um sistema transistorizado que chaveia a bomba;

3 Depois é produzida uma vazão variável proporcional ao nível de tensão informado pelo CLP.

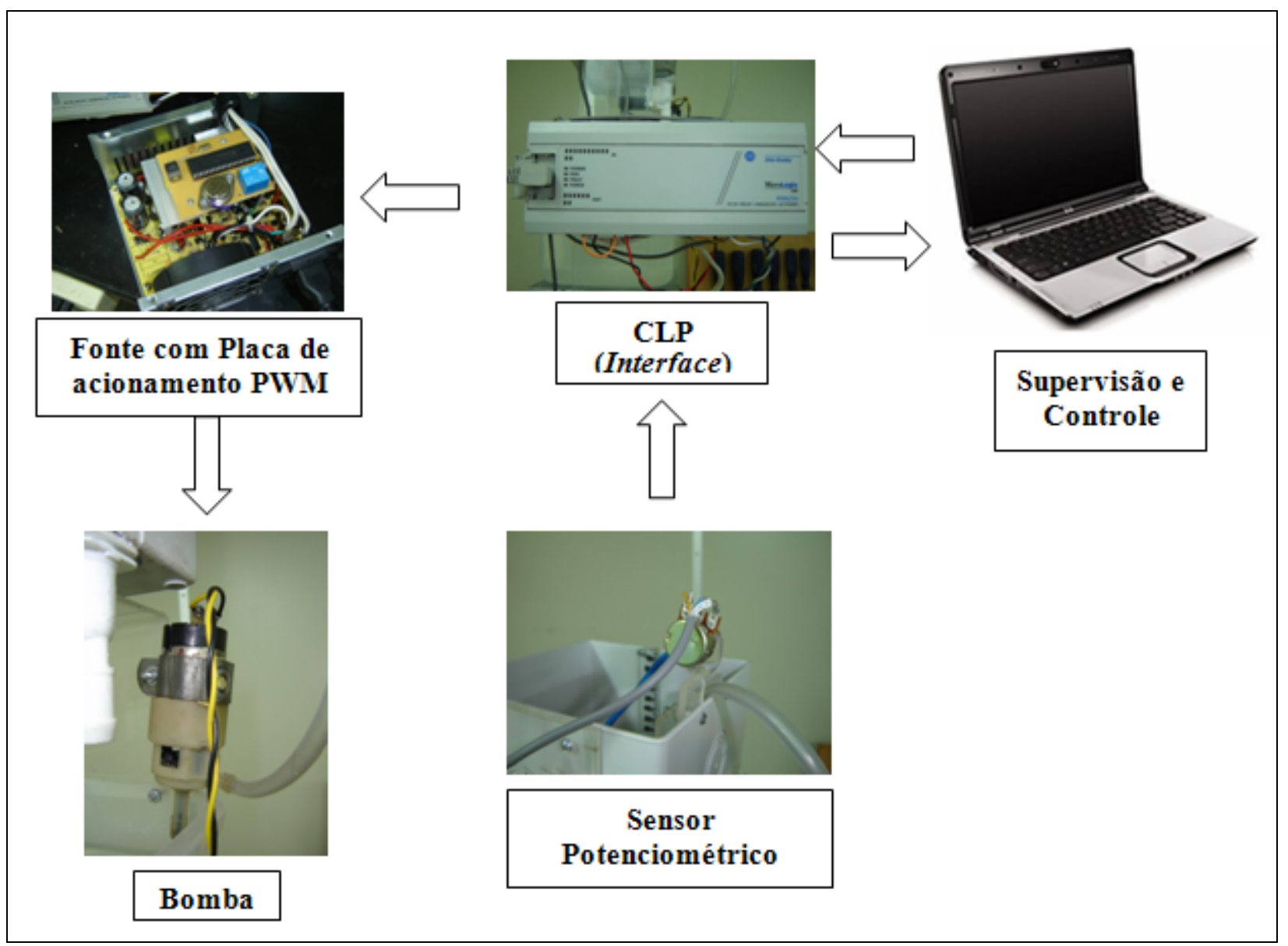

Figura 1 - Visão geral dos elementos integrantes do kit

Todo o sistema é operado e intertravado pelo aplicativo supervisório. Nesse aplicativo, são mostrados ao usuário os valores de todas as variáveis do sistema, bem como, os dados da bomba, alarmes, gráficos de tendência, além de todos os parâmetros do controlador didático. O sistema também realiza as operações matemáticas do controlador no aplicativo de supervisão enviando em seguida esse resultado para o CLP, que por sua vez irá informar a bomba à velocidade necessária que ela deve atingir para que o nível desejado seja alcançado.

Visto em diagrama de blocos o sistema consta de um sistema clássico de controle aplicando a técnica PI (Proporcional Integral), como pode ser visto na Figura 2. Na Figura 3 é possível observar o kit montado, que atualmente está sendo utilizado nas aulas de controle de processos. 


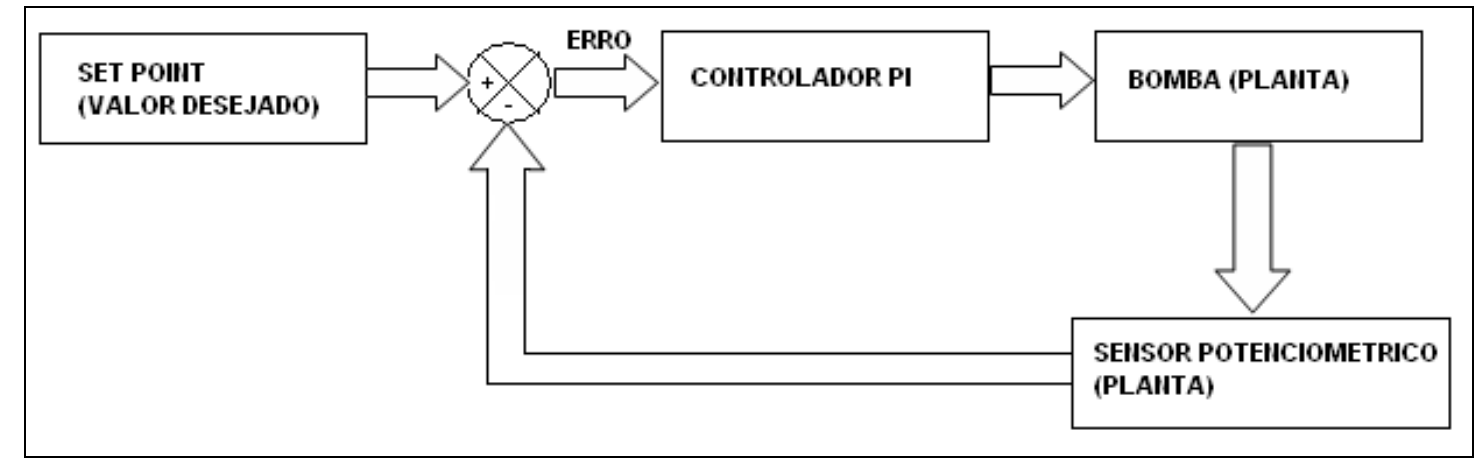

Figura 2 - Diagrama de blocos que representa o sistema

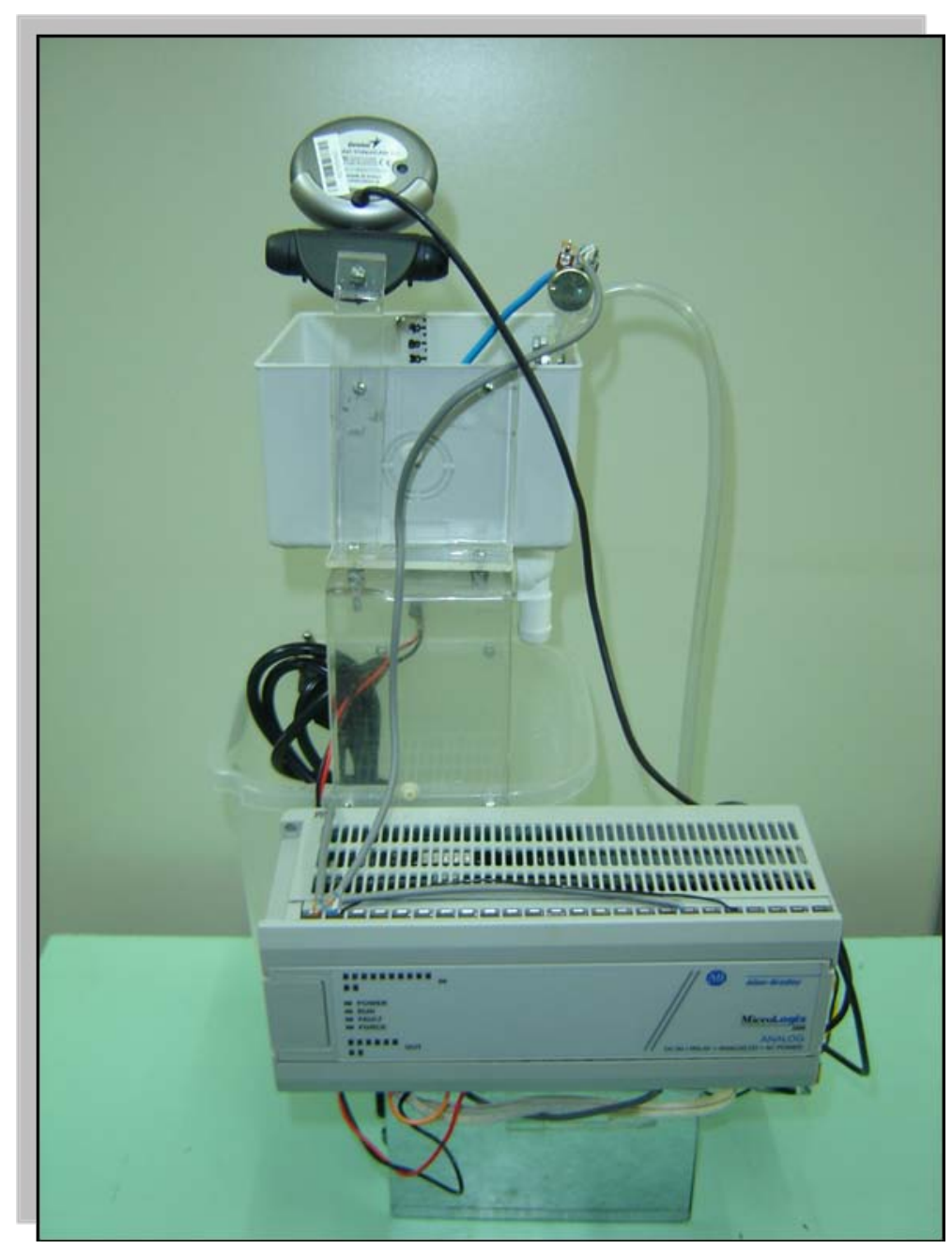

Figura 3 - Kit educacional para controle e supervisão aplicado de nível de fluido. 


\section{CONSIDERAÇÕES FINAIS}

De uma maneira geral, pode-se considerar que as atividades desenvolvidas durante o projeto e implementação do kit didático para o estudo de controladores PID aplicados a um sistema de primeira ordem para controle de nível foram bem sucedidas. Em decorrência do reflexo observado pelo bom desempenho dos alunos em cursos de sistemas de controle e automação industrial onde se fez uso do kit.

Pode-se ressaltar também que os dois requisitos de projeto que foram atingidos: o primeiro deles relativo ao baixo custo final do equipamento e o segundo referente a portabilidade do mesmo que possibilita seu transporte para cursos e treinamentos realizados fora do ambiente de laboratórios acadêmicos.

\section{REFERÊNCIAS BIBLIOGRÁFICAS}

1. $\quad$ ELONKA, Stephen M e Par, Alonzo R. Manual de instrumentação. Vol 1.

2. http://www.ene.unb.br/ gaborges/disciplinas/ca/seminarios/2005.1/inversores.doc. Arquivo capturado em 11 de julho de 2006.

3. RIBEIRO, Marco Antônio. Apostila do SENAI -ES.

4. SILVA, Walmy André Cavalcante Melo. Aula 04, Visão Geral do Protocolo Modbus. Disponível na internet via WWW. URL: http://www.cefetrn.br/ walmy/RI_A4.pdf. Capturado em 19 de julho de 2006. 